\title{
Proportional-Integral-Derivative-Based Learning Control for High-Accuracy Repetitive Positioning of Frictional Motion Systems
}

Citation for published version (APA):

Hazeleger, L., Beerens, R., \& van de Wouw, N. (2021). Proportional-Integral-Derivative-Based Learning Control for High-Accuracy Repetitive Positioning of Frictional Motion Systems. IEEE Transactions on Control Systems Technology, 29(4), 1652-1663. [9179755]. https://doi.org/10.1109/TCST.2020.3017803

Document license:

TAVERNE

DOI:

10.1109/TCST.2020.3017803

Document status and date:

Published: 01/07/2021

Document Version:

Publisher's PDF, also known as Version of Record (includes final page, issue and volume numbers)

\section{Please check the document version of this publication:}

- A submitted manuscript is the version of the article upon submission and before peer-review. There can be important differences between the submitted version and the official published version of record. People interested in the research are advised to contact the author for the final version of the publication, or visit the DOI to the publisher's website.

- The final author version and the galley proof are versions of the publication after peer review.

- The final published version features the final layout of the paper including the volume, issue and page numbers.

Link to publication

\footnotetext{
General rights

- You may freely distribute the URL identifying the publication in the public portal. follow below link for the End User Agreement:

www.tue.nl/taverne

\section{Take down policy}

If you believe that this document breaches copyright please contact us at:

openaccess@tue.nl

providing details and we will investigate your claim.
}

Copyright and moral rights for the publications made accessible in the public portal are retained by the authors and/or other copyright owners and it is a condition of accessing publications that users recognise and abide by the legal requirements associated with these rights.

- Users may download and print one copy of any publication from the public portal for the purpose of private study or research.

- You may not further distribute the material or use it for any profit-making activity or commercial gain

If the publication is distributed under the terms of Article 25fa of the Dutch Copyright Act, indicated by the "Taverne" license above, please 


\title{
Proportional-Integral-Derivative-Based Learning Control for High-Accuracy Repetitive Positioning of Frictional Motion Systems
}

\author{
Leroy Hazeleger ${ }^{(}$, Member, IEEE, Ruud Beerens $^{(}$, and Nathan van de Wouw ${ }^{\circledR}$, Senior Member, IEEE
}

\begin{abstract}
Classical proportional-integral-derivative (PID) control is exploited widely in industrial motion systems with dry friction motivated by the intuitive and easy-to-use design and tuning tools available. However, classical PID control suffers from severe performance limitations. In particular, friction-induced limit cycling (i.e., hunting) is observed when integral control is employed on frictional systems that suffer from the Stribeck effect, thereby compromising setpoint stability. In addition, the resulting time-domain behavior, such as rise time, overshoot, settling time, and positioning accuracy, highly depends on the particular frictional characteristic, which is typically unknown or uncertain. On the other hand, omitting integral control can lead to constant nonzero setpoint errors (i.e., stick). To achieve superior setpoint performance for frictional motion systems in a repetitive motion setting, we propose a PID-based feedback controller with a time-varying integrator gain design. To ensure optimal setpoint positioning accuracy, a data-based sampled-data extremum-seeking architecture is employed to obtain the optimal time-varying integrator gain design. The proposed approach does not rely on knowledge on the friction characteristic. Finally, the effectiveness of the proposed approach is evidenced experimentally by application to an industrial nanopositioning motion stage setup of a high-end electron microscope.
\end{abstract}

Index Terms-Frictional motion systems, iterative learning control (ILC), repetitive motion, sampled-data extremum seeking, Stribeck friction.

\section{INTRODUCTION}

$\mathbf{M}$ ANY industrial motion systems perform repetitive tasks, e.g., repetitive motion profiles in pick-and-place machines [1, Sec. 5], large-scale transferring of circuit topology to silicon wafers in lithography systems [2], and automated scanning procedures in electron microscopes. Due to demands on hardware cost reduction in the design phase or

Manuscript received September 13, 2019; revised May 25, 2020; accepted July 29, 2020. Date of publication August 28, 2020; date of current version June 10, 2021. Manuscript received in final form August 17, 2020. This work was supported by the Netherlands Organisation for Scientific Research (NWO) through the CHAMeleon Research Programme under Project 13896. Recommended by Associate Editor K. Barton. (Corresponding author: Leroy Hazeleger.)

Leroy Hazeleger and Ruud Beerens are with the Department of Mechanical Engineering, Eindhoven University of Technology, 5600 MB Eindhoven, The Netherlands (e-mail: 1.hazeleger@tue.nl; r.beerens@tue.nl).

Nathan van de Wouw is with the Department of Mechanical Engineering, Eindhoven University of Technology, 5600 MB Eindhoven, The Netherlands, and also with the Department of Civil, Environmental and GeoEngineering, University of Minnesota, Minneapolis, MN 55455 USA (e-mail: n.v.d.wouw@tue.nl).

Color versions of one or more of the figures in this article are available online at https://ieeexplore.ieee.org.

Digital Object Identifier 10.1109/TCST.2020.3017803 wear in the operational phase, friction is commonly present in such high-precision positioning systems, thereby limiting the achievable positioning accuracy.

Various control solutions have been presented throughout the literature to cope with frictional effects in motion systems. Model-based compensation techniques (see [3], [4]) exploit parametric models in the control loop to compensate for friction. However, as friction characteristics are commonly unknown, uncertain, and (slowly) time-varying, model-based methods are prone to modeling errors, ultimately compromising positioning performance. Nonmodel-based methods, e.g., impulsive control (see [5]), dithering-based techniques (see [6]), and sliding-mode control (see [7]), may result in stability of the setpoint. In general, these nonmodel-based control techniques have a common disadvantage. Namely, the persistent injection of high-frequency control signals may excite unmodeled high-frequency system dynamics, which is highly undesirable in motion systems, and therefore, these techniques are not appealing for industrial applications.

Despite the existence of the abovementioned control techniques, the vast majority of the high-precision industry still employs classical proportional-integral-derivative (PID) control since control practitioners are often well-trained in linear control design (loop shaping). Moreover, it is well known that the integral action in PID control is capable of compensating for unknown static friction in motion systems (see [8]-[10]). However, PID control is prone to performance limitations as well. For example, solutions settle on a persistent oscillation around the setpoint when the integral control is employed on systems where the friction characteristic includes the velocity-weakening (Stribeck) effect so that stability of the setpoint is not achieved (this phenomenon is also called friction-induced limit cycling or hunting (see [11] and Section II-A). Even if stability can be warranted, rise time, overshoot, settling time (see [9]), and positioning accuracy depend on the particular friction characteristic, which is highly uncertain in practice. Hence, despite the popularity of the PID controller in industry, friction is a performance- and reliability-limiting factor in PID-controlled motion systems. This motivates the development of a more advanced control strategy while preserving the benefits and intuition of classical PID feedback control design.

In this article, we propose a PID-based learning controller in order to achieve a high setpoint accuracy for repetitive 
tasks in motion systems subject to unknown static and velocity-dependent friction, including the Stribeck effect. The PID-based learning controller consists of two elements. First, a PID control architecture with a time-varying integrator gain design is proposed, facilitating a tailored design for the repetitive motion and friction characteristic at hand. In this manner, friction-induced limit cycles can be avoided, and high accuracy repetitive setpoint positioning and improved transient behavior can be achieved instead. In addition, similar robustness properties as classical PD control at the desired setpoint can be achieved. Second, we propose a data-driven, model-free optimization strategy, in order to iteratively find the optimal time-varying integrator gain, in the presence of unknown friction. Such a data-driven tuning procedure yields optimal setpoint accuracy and improved transient behavior.

In this work, the finite-horizon optimization problem of finding the optimal time-varying integrator gain for repetitive motion tasks is formulated in terms of a model-free sampled-data extremum-seeking control (ESC) problem (see [12]-[14]). This is achieved by exploiting a linear spline basis function parameterization of the time-varying integrator gain. The extremum-seeking mechanism is then designed to iteratively improve system performance by adaptive tuning of the parameters of this basis function parameterization. This learning mechanism has resemblance with iterative feedback tuning (see [15]-[17]) and iterative learning control (ILC ([18], [19]). In a linear motion control setting, these methods have proven merit. However, for our nonlinear setting, employing an extremum-seeking approach instead is beneficial. Namely, ESC is able to deal with unknown, uncertain, time-varying, and general nonlinear systems and is therefore suitable to be used in the presence of unknown nonlinear frictional effects. Moreover, the potential of ESC in the context of ILC and optimizing transient behavior has been shown in, e.g., [20]-[23]. However, it must be noted that the extremum-seeking strategy to iteratively improve the system's transient behavior proposed in this work is different. Namely, we iteratively learn timevarying feedback controller gains using ESC, where, e.g., in [22] and [21], ESC is used for iterative tuning of PID controllers having constant gains, and in, e.g., in [20] and [23], (sampled-data) ESC is employed to iteratively tune a system input signal.

The main contributions of this article can be summarized as follows. The first contribution is a parameterized time-varying integrator gain design for frictional motion systems. The second contribution is an automatic controller tuning procedure based on a sampled-data extremum-seeking framework. The third contribution is an experimental case study on an industrial high-precision motion stage of an electron microscope. This article builds upon preliminary results presented in [24], which contains a particular discontinuous integrator gain design and merely simulation examples. In addition to [24], this article contains a more generic controller structure, a novel parameterization of the integrator gain that leads to a continuous control signal (which is highly desirable in practice), a formal analysis of the existence of a static input-output map of the closed-loop system (allowing for the use of ESC), and an extensive experimental case study.

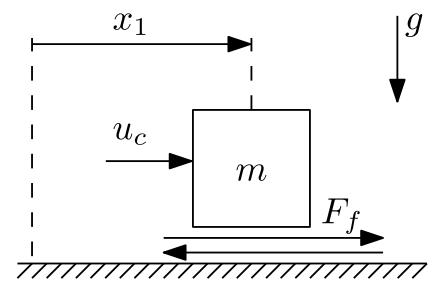

Fig. 1. Schematic of the motion system subject to a friction force $F_{f}$.

The remainder of this article is organized as follows. We formalize the control problem in Section II, and we present the PID-based controller with time-varying integrator gain in Section III. In Section IV, we present the extremum-seekingbased iterative learning mechanism. Section V provides an implementation summary. In Section VI, we experimentally show the working principles of the proposed PID-based learning controller, applied to an industrial nanopositioning motion stage. Conclusions are presented in Section VII.

Notation: Sign(.) (with an upper case S) denotes the setvalued sign function, i.e., $\operatorname{Sign}(y):=1$ for $y>0, \operatorname{Sign}(y):=$ -1 for $y<0$, and $\operatorname{Sign}(y):=[-1,1]$ for $y=0$. $\mathbb{B}$ denotes the closed unit ball of appropriate dimensions, in the Euclidean norm.

\section{Control Problem Formulation For Frictional MOTION SySTEMS}

In this section, we first present a PID-controlled motion system with (Stribeck) friction, to illustrate the shortcomings of $\mathrm{P}(\mathrm{I}) \mathrm{D}$ control for frictional motion systems. Second, we state the control problem formulation for repetitive positioning of frictional motion systems.

\section{A. PID-Controlled Single Mass System With Stribeck Friction}

Consider a single-degree-of-freedom motion system, consisting of a mass $m[\mathrm{~kg}]$ sliding on a horizontal plane, with measurable position $x_{1}[\mathrm{~m}]$, velocity $x_{2}[\mathrm{~m} / \mathrm{s}]$, and control input $u_{c}[\mathrm{~N}]$ (i.e., the actuation force as determined by a motion control algorithm). The mass is subject to a friction force $F_{f}$ (as schematically shown in Fig. 1) belonging to a friction set $\Phi\left(x_{2}\right)$ for a velocity $x_{2}$, where $x_{2} \rightrightarrows \Phi\left(x_{2}\right)$ is a set-valued mapping. The set-valued friction characteristic $\Phi$ consists of a Coulomb friction component with (unknown) static friction $F_{s}$, a viscous contribution $\gamma x_{2}$ (where $\gamma \geq 0$ is the viscous friction coefficient), and a nonlinear velocity-dependent friction component $f$, encompassing the Stribeck effect, i.e.,

$$
F_{f} \in \Phi\left(x_{2}\right):=-F_{s} \operatorname{Sign}\left(x_{2}\right)-\gamma x_{2}+f\left(x_{2}\right) .
$$

We pose the following assumption on the velocity-dependent friction component $f$.

Assumption 1: The function $f: \mathbb{R} \rightarrow \mathbb{R}$ is continuously differentiable and satisfies the following.

1) $|f(v)| \leq F_{s}$ for all $v$.

2) $v f(v) \geq 0$ for all $v$.

3) $f$ is globally Lipschitz with Lipschitz constant $L>0$. 
The dynamics are governed by the following differential inclusion:

$$
\begin{aligned}
\dot{x}_{1} & =x_{2} \\
m \dot{x}_{2} & \in \Phi\left(x_{2}\right)+u_{c} .
\end{aligned}
$$

Let us consider a classical PID controller for input $u_{c}$ in (2), i.e.,

$$
\begin{aligned}
& u_{c}=k_{p} e+k_{d} \dot{e}+k_{i} x_{3} \\
& \dot{x}_{3}=e
\end{aligned}
$$

where $e:=r-x_{1}$ denotes the setpoint error with $r$ the reference signal, $x_{3}$ the integrator state, and $k_{p}, k_{d}$, and $k_{i}$ the proportional, derivative, and integral controller gains, respectively. For frictional motion systems, the presence of an integrator action in (3) is motivated by the fact that it is able to compensate for unknown static friction, due to the buildup of control force by integrating the position error. In general motion control systems, integrator action is widely used to improve low-frequency disturbance rejection properties and shorten rise times, the latter being beneficial for machine throughput. As an illustration, consider a constant reference $r$, i.e., a point-to-point motion so that the resulting set of equilibria of closed-loop system (1)-(3) is given by

$$
\mathcal{E}_{\text {pid }}=\left\{\left(e, \dot{e}, x_{3}\right) \in \mathbb{R}^{3}|e=0, \dot{e}=0,| x_{3} \mid \leq F_{s} / k_{i}\right\}
$$

which is globally asymptotically stable for closed-loop system dynamics (1)-(3) only when $f(\cdot)=0$, i.e., in the absence of the velocity-weakening (Stribeck) effect (see [8]). In the presence of the Stribeck effect, however, $\mathcal{E}_{\text {pid }}$ is not stable and limit cycling (hunting) occurs. Although static friction is eventually compensated by the integrator action, friction is overcompensated in the slip phase that follows due to the velocity-weakening effect, resulting in overshoot of the setpoint. This process repeats and results in stick-slip limit cycling, compromising setpoint stability. This phenomenon is illustrated experimentally in the third subplot in Fig. 2. In particular, Fig. 2 shows the reference trajectory employed (subplot 1), the measured error response when using solely PD control (subplot 2), two measured error responses using PID control (subplot 3), and the corresponding control forces generated by the PD and PID controllers (subplot 4) of an industrial nanopositioning motion stage setup (the motion stage setup will be discussed in more detail in Section VI). A relatively large positioning error of about $200 \mathrm{~nm}$ is obtained when using PID control. Omission of the integrator action [i.e., PD control for input $u_{c}$ in (2)] results in the set of equilibria for (1)-(3) given by

$$
\mathcal{E}_{p d}=\left\{(e, \dot{e}) \in \mathbb{R}^{2}|| e \mid \leq F_{s} / k_{p}, \dot{e}=0\right\}
$$

which is stable (see [10]), but does not guarantee zero steady-state error, as shown in subplot 2 of Fig. 2. In particular, the size of the achievable steady-state error depends inversely on the proportional gain $k_{p}$, which cannot be chosen arbitrarily large for stability purposes. The drawbacks of P(I)D control for frictional motion systems motivate the design of a more suitable control architecture. Respecting the popularity of PID control in industry, we propose a time-varying PID-based
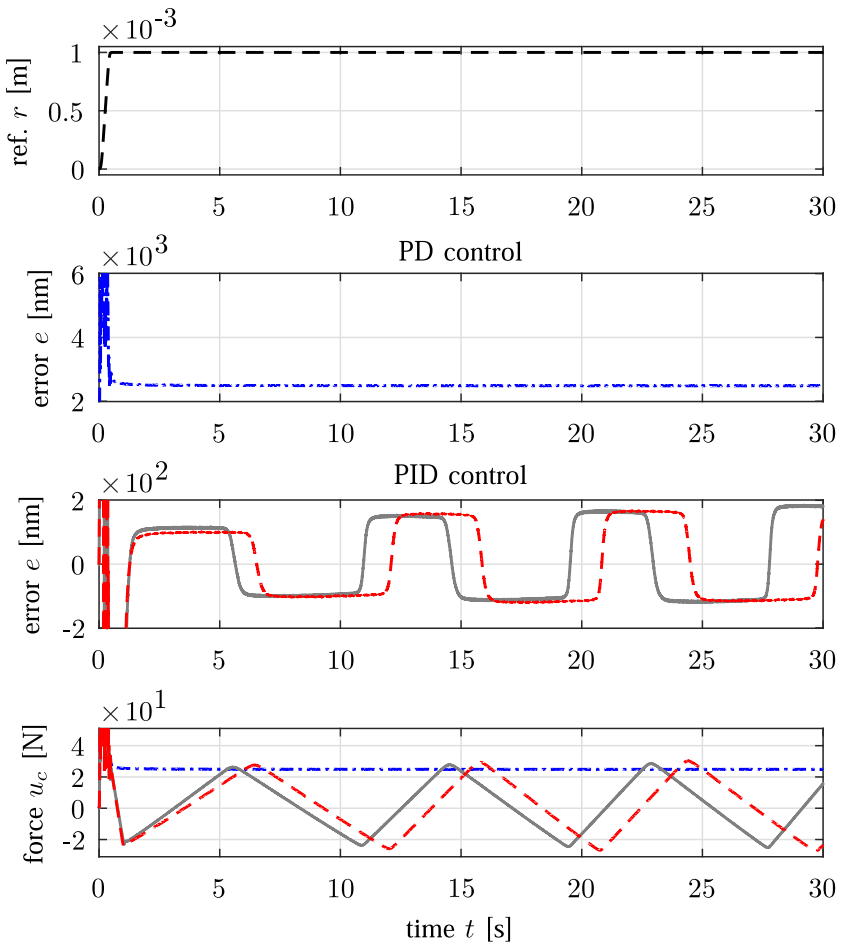

Fig. 2. Measured error responses of an industrial nanopositioning motion stage setup subject to a third-order reference trajectory ( - -), for details about the set-up, see Section VI, and the corresponding control forces of a PD controller (--) , and a PID controller with fixed integrator gain $k_{i}=1 \times 10^{8}$ ( - ) and ( --$)$. The PD controller yields a nonzero steadystate positioning error, and the PID controller induces hunting.

controller in Section III after formalizing the control problem in Section II-B.

\section{B. Control Problem Formulation}

In this article, we focus on achieving high-accuracy positioning for frictional motion systems that perform a $T$ - repetitive motion. We consider, for the position $x_{1}$, a desired repetitive reference $r$, defined on the time interval $[0, T]$, where the system starts and ends at rest. Specifically, we separate the time interval $[0, T]$ into two particular parts, which are specified as follows.

1) $t \in\left[0, T_{B}\right)$; the so-called transient time window, during which the system is allowed to move from 0 to $r$.

2) $t \in\left[T_{B}, T\right]$; the so-called standstill time window, during which standstill at $r$ is required. The time interval $\left[T_{B}, T\right]$ is typically used by the industrial machine, of which the motion system is part, to perform a certain machining operation, for which accurate positioning is required.

Respecting the popularity of PID control in industry, and considering the advantages of classical P(I)D control, in this article, we address the following setpoint control problem.

Problem 1: Design a PID-based control strategy for motion systems of the form (1) and (2) that perform a repetitive motion profile and are subject to unknown static and velocity-dependent friction such that high-accuracy setpoint positioning during the standstill time window is achieved and 
optimal transient behavior during the transient time window is achieved.

The desired performance, i.e., an optimal transient response on $\left[0, T_{B}\right)$ and optimal setpoint accuracy on $\left[T_{B}, T\right]$, can be captured by the following cost function $J$ to be minimized:

$$
J(e):=\int_{0}^{T}|w(t) e(t)|^{2} d t
$$

where we have introduced a weighting function $w(t)$ that is defined as follows:

$$
w(t):= \begin{cases}w_{1} & \text { if } t \in\left[0, T_{B}\right) \\ w_{2} & \text { if } t \in\left[T_{B}, T\right]\end{cases}
$$

with $w_{1}, w_{2} \in \mathbb{R}$ suitable weighting factors, trading off the emphasis on transient performance versus setpoint accuracy. Other (transient) performance relevant variables, such as the control effort $u_{c}$, or the velocity $x_{2}$ of the mass can be considered in (6) as well, if accurate velocity measurements are available.

\section{Time-VARYing Integrator GAIN Design FOR PID-BASEd Control of Frictional Motion Systems}

In this section, first, the time-varying integrator design is presented, and subsequently, the achievable performance benefits are shown in a numerical example.

\section{A. Time-Varying Integrator Gain Design}

The limit cycle present in the case of PID control with constant integrator gain (see Fig 2) is caused by the buildup of integrator action (during transients and the stick phase) in interplay with the friction characteristic. This observation motivates the design of a novel time-varying integrator gain $k_{i}(t)$ for point-to-point motion for the following reasons.

1) The presence of integrator action still allows the system to escape undesired stick phases.

2) Overcompensation of friction due to, e.g., a severe Stribeck effect, can be avoided, by altering $k_{i}(t)$ during the slip phase.

3) Zero integral action can be enforced at the setpoint when standstill of the system is required such that robustness against other force disturbances is provided by the static friction.

The resulting controller is then given by

$$
\begin{aligned}
& u_{c}=k_{p} e+k_{d} \dot{e}+k_{i}(t) x_{3} \\
& \dot{x}_{3}=\varsigma(t) e
\end{aligned}
$$

with $\varsigma(t) \in\{0,1\}$ a to-be-designed switching function that prevents uncontrolled growth of $x_{3}$. Furthermore, the to-bedesigned time-varying integral gain $k_{i}(t)$ should be bounded, i.e., $\left|k_{i}(t)\right|<+\infty$ for all $t \in[0, T]$. Here, we opt to employ a time-varying integrator gain, instead of an appropriate feedforward control signal in combination with a constant integrator gain as commonly done in ILC to, e.g., counteract recurring disturbances [18], [19]. This choice is motivated by the fact that with the proposed controller, we are able to escape undesired stick phases by enabling $k_{i} \neq 0$ during the transient time window and create robustness to other force disturbances close to the setpoint, by enforcing $k_{i}=0$ during the standstill time window. Integrator action is then disabled so that the system remains in standstill since buildup of control force is prevented.

Remark 1: Note that the presented engineering intuition here only applies when the integrator gain $k_{i}$ is placed at the right-hand side in (8a), instead of at the right-hand side in (8b). Indeed, in the latter case, $k_{i}=0$ would still yield a constant integral control force in $u_{c}$.

We now propose a parametric design for $k_{i}(t)$, parameterized by a finite set of basis functions $\varphi^{(j)}, j \in\{1,2, \ldots, b\}$, as follows:

$$
k_{i}(t):=\sum_{j=1}^{b} \varphi^{(j)}(v, t)
$$

where $b$ denotes the number of basis functions and $v \in \mathbb{R}^{n_{v}}$ is a to-be-designed parameter vector. Next, we give two examples of basis function parameterizations that can be employed to facilitate solving problem 1

Example 1 [24]: Steplike basis functions, i.e., $\varphi^{(j)}(v, t):=v^{(j)} \Psi^{(j)}(t)$ with $\Psi^{(j)}(t)$ defined as follows:

$$
\Psi^{(j)}(t):=\left\{\begin{array}{ll}
1, & t \in\left[(j-1) t_{s}, j t_{s}\right) \\
0, & t \notin\left[(j-1) t_{s}, j t_{s}\right)
\end{array} \text { for } j=1, \ldots, b\right.
$$

where $t_{s}$ satisfies $T=b t_{s}$, and the to-be-designed parameter vector $v \in \mathbb{R}^{n_{v}}$, with $n_{v}=b$.

Example 2: Linear spline basis functions, i.e., $\varphi^{(j)}(v, t):=$ $\left[v^{(j)} v^{(j+1)}\right] \Psi^{(j)}(t)$ with $\Psi^{(j)}(t)$ defined as follows:

$$
\Psi^{(j)}(t):=\left\{\begin{array}{lr}
{\left[\begin{array}{cc}
1-\frac{t-(j-1) t_{s}}{t_{s}} & \frac{t-(j-1) t_{s}}{t_{s_{s}}}
\end{array}\right],} & t \in\left[(j-1) t_{s}, j t_{s}\right) \\
{\left[\begin{array}{ll}
0 & 0
\end{array}\right]^{T_{s},}} & t \notin\left[(j-1) t_{s}, j t_{s}\right) \\
& \text { for } j=1, \ldots, b
\end{array}\right.
$$

where $t_{s}$ satisfies $T=b t_{s}$, and the parameter vector $v \in \mathbb{R}^{n_{v}}$, with $n_{v}=b+1$.

Remark 2: Other types of basis function designs can be adopted from the ILC literature. For example, polynomial bases (see [25], [26]) and rational bases (see [27]) can similarly be exploited.

In the remainder of this article, we opt for a linear spline basis function parameterization of $k_{i}(t)$, as illustrated in Example 2, as it yields a continuous control signal. In contrast, the steplike basis function parameterization, as illustrated in Example 1 and used in [24], results in discontinuities in the control signal, risking excitation of high-frequency system dynamics, which is also a well-known problem in reset control and impulsive control strategies developed for the control of frictional systems.

The switching function $\varsigma(t)$ in (8) is analogously designed as

$$
\varsigma(t):= \begin{cases}1, & t \in\left[0, T_{B}\right) \\ 0, & t \in\left[T_{B}, T\right]\end{cases}
$$


so that the evolution of the integrator state is disabled on the interval $\left[T_{B}, T\right]$. Summarizing, the resulting closed-loop system with the proposed design for the time-varying integrator gain is given by (1), (2), (8), (9), (11), and (12).

Let us now present some properties of the resulting closed-loop system, which will be instrumental in the data-based sampled-data extremum-seeking architecture presented in Section IV. In particular, the following proposition asserts that each bounded realization of $k_{i}(t)$ results in a unique solution $x=\left(x_{1}, x_{2}, x_{3}\right)^{\top}$ of the closed-loop system, which is bounded on the interval $[0, T]$.

Proposition 1: Under Assumption 1, for any constant $r$, each bounded realization of $k_{i}(t)$ in (8) satisfying $k_{i}(t)=0$ for $t \in\left[T_{B}, T\right]$, each initial condition satisfying $x(0) \in K_{1} \mathbb{B}$ with $K_{1} \geq 0$, and $\varsigma(t)$ as in (12), solutions $x(t)$ to closed-loop system (1), (2), (8), (9), (11), and (12) are unique and satisfy $x(t) \in K_{2} \mathbb{B}$ for some bounded $K_{2}>0$, for all $t \in[0, T]$.

Proof 1: See the Appendix.

\section{B. Illustrative Example}

We illustrate the potential of the proposed time-varying integrator gain by means of a numerical example. Consider closed-loop system (1), (2), (8), (9), (11), and (12), where we adopt the following numerical values: $m=1, k_{p}=18 \mathrm{~N} / \mathrm{m}$, $k_{d}=2 \mathrm{Ns} / \mathrm{m}, F_{s}=0.981 \mathrm{~N}$, and $\gamma=0.5$. The Stribeck contribution of the friction $f$ is given by

$$
f\left(x_{2}\right)=\left(\left(F_{s}-F_{c}\right) \eta x_{2}\right)\left(1+\eta\left|x_{2}\right|\right)^{-1}
$$

where $F_{c}$ is the Coulomb friction force and $\eta$ is the Stribeck shape parameter. The motion profile interval is characterized by $T=1.5 \mathrm{~s}$ and $T_{B}=0.75 \mathrm{~s}$. For the time-varying integrator gain design, we take $b=6$, and the parameter vector $v$ is given by $v=\left[\begin{array}{lllllll}v^{(1)} & v^{(2)} & v^{(3)} & 0 & 0 & 0 & 0\end{array}\right]^{\top}$. Moreover, $v^{(1)}$ is kept fixed to $v^{(1)}=25 \mathrm{~N} /(\mathrm{ms})$, which enables integral action at least for $t \in\left[0, t_{s}\right)$ to escape from a potential initial stick phase. The parameters $v^{(2)}$ and $v^{(3)}$ are tunable and affect the error response of the closed-loop system, as illustrated next.

We illustrate the potential performance benefits of the controller by considering two cases with different friction characteristics: 1) $F_{c}=F_{s} / 2, \eta=20$, and $\gamma=0$ and 2) $F_{c}=F_{s} / 3, \eta=60$, and $\gamma=1$. Consider Fig. 3, where, for each column, the top subplot depicts the friction characteristic, the middle subplot depicts four different position error evolutions, and the bottom subplot depicts the four corresponding designs for the time-varying integrator gain $k_{i}(t)$. For both friction cases, the error response with a classical PID controller (i.e., with a fixed integrator gain $k_{i}=25 \mathrm{~N} /(\mathrm{ms})$ and depicted by (-.-)) leads to significant overshoot and eventually limit cycling (the latter explicitly visible for case 2 , see also Fig. 2 for such limit-cycling effect). We now perform three different simulations for both cases by selecting different pairs of values for the parameters $v^{(2)}$ and $v^{(3)}$, which yield different error responses. The first simulation in friction case $1(-)$ results in significant overshoot, and the second simulation in friction case $1(--)$ results in significant undershoot. The optimal choice for $v^{(2)}$ and $v^{(3)}$ instead results in zero error in friction case 1 , see the third simulation $(-)$. Note that the friction
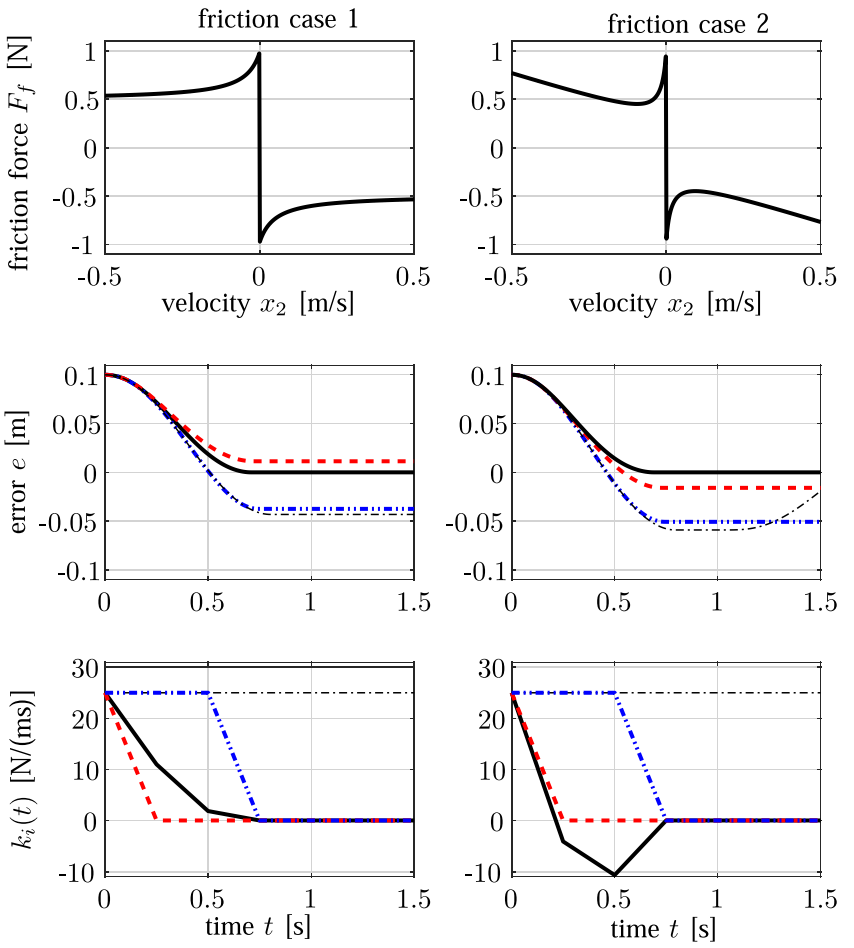

Fig. 3. Simulation results, where each column presents from top to bottom the friction characteristic, error response, and time-varying integrator gain for cases 1 and 2, respectively. The different error responses in the middle subplots correspond to simulations with different realizations of the time-varying integrator gain as in the lower subplots, indicated by corresponding colors and line styles. In addition, the classical PID responses with constant $k_{i}$ are indicated by $(-\cdot-)$.

characteristic in case 2 has a more severe Stribeck effect compared with the characteristic in case 1 (see the top subplot), whereby the optimal settings for $v^{(2)}$ and $v^{(3)}$ become negative, but zero steady-state error is still achieved, see ( - ) in the second and lower subplots. The proposed time-varying PID controller is hence capable of achieving optimal positioning performance, despite the presence of friction, by proper tuning of the parameters in $v$.

Since the friction characteristic $\Phi$ in (2) is generally unknown and uncertain and can change (slowly) in time, the optimal design for the tunable parameters in $v$ is challenging or even impossible using a model-based approach only. Therefore, we propose a data-based extremum-seeking-based (learning) algorithm in Section IV, to learn the optimal $k_{i}(t)$ by adaptive tuning of the parameter vector $v$, on the basis of measured error responses.

\section{SAmpled-Data Extremum Seeking for Iterative LEARNING IN RePETITIVE SETPOINT Positioning}

\section{A. Sampled-Data Extremum-Seeking Framework}

In this section, we propose a sampled-data extremumseeking strategy, akin to ILC, to optimize the time-varying integrator gain design presented in Section III to achieve high-accuracy setpoint positioning. Specifically, given the cost function in (6) and (linear spline) basis function parameterization of the time-varying integrator gain in (9), we can formulate the finite-horizon optimization problem as a model-free 


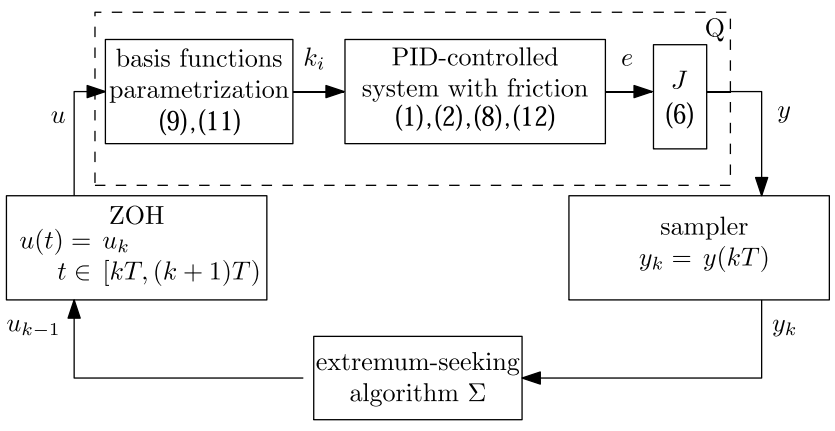

Fig. 4. Sampled-data extremum-seeking framework based on sampled-data control law with periodic sampling time $T$, and sampler and zero-order hold elements.

sampled-data extremum-seeking problem (see [14], [20]). Namely, consider the cascade connection of the PID-controlled motion system given by (1), (2), (8), (9), (11), and (12) and the cost function $J$ in (6). In addition, we consider the tobe-designed parameter vector to be decomposed as follows: $v=v_{0}+C u$, where $v_{0} \in \mathbb{R}^{n_{v}}$ is a user-defined parameter vector, $C \in \mathbb{R}^{n_{0} \times p}$ is a user-defined selection matrix, and $u \in \mathbb{R}^{p}$ is the vector of to-be-optimized parameters by the extremum-seeking algorithm. This cascade connection yields the following unknown static input-output map $Q: \mathbb{R}^{p} \rightarrow \mathbb{R}$ for the cascaded system (1), (2), (6), (8), (9), (11), and (12):

$$
Q(u):=\int_{0}^{T}|w(t) e(t)|^{2} d t
$$

where the weighting function $w(t)$ is defined in (7). It must be noted that periodic reinitialization of the states to fixed values, in combination with Proposition 1, is needed for ESC to be applicable in an iterative learning context, i.e., $x(k T)=x_{0}$ for all $k=1,2, \ldots$ Only under these conditions (reinitialization and uniqueness provided by Proposition 1), $Q$ in (14) is uniquely defined (see also Remark 4). In addition, the fact that solutions to the closed-loop system remain bounded by Proposition 1 guarantees that $Q(u)$ is bounded.

Based solely on output measurements, which we use to compute $Q$ in (14), ESC is exploited to adaptively find parameters $u$ that minimize $Q$. Fig. 4 schematically shows the sampled-data extremum-seeking framework, i.e., the interconnection of the PID-based controlled frictional motion system with a basis function parametrization (1), (2), (8), (9), (11), and (12) and the cost function $J$ in (6) implemented as follows:

$$
y(t):=J(e(t))=\int_{t-T}^{t}|w(s) e(s)|^{2} d s
$$

where $e(s)=0$ for $s \in[-T, 0)$ and with the weighting function implemented as follows:

$$
w(t):= \begin{cases}w_{1} & \text { if } \bmod (t, T) \in\left[0, T_{B}\right) \\ w_{2} & \text { if } \bmod (t, T) \in\left[T_{B}, T\right]\end{cases}
$$

with a $T$-periodic sampler, a discrete-time extremum-seeking algorithm $\Sigma$, and a zero-order hold ( $\mathrm{ZOH})$ element. Let $\left\{u_{k}\right\}_{k=0}^{\infty}$ be a sequence of vectors generated by the extremum-seeking algorithm $\Sigma$ based on collected measurements, and define the $\mathrm{ZOH}$ operation as follows:

$$
u(t):=u_{k} \quad \forall t \in[k T,(k+1) T)
$$

with $k=0,1,2, \ldots$, and sampling period $T>0$ with $u_{k} \in \mathbb{R}^{p}$, for all $k$. Let us define the ideal periodic sampling operation

$$
y_{k}:=y(k T), \quad k=1,2, \ldots
$$

where $y_{k}=Q\left(u_{k-1}\right)$ are the collected measurements as used by the extremum-seeking algorithm $\Sigma$ (see Fig. 4). We care to stress that $T$ is the sampling period of the extremum-seeking controller, which conforms to the period time of the motion profile, and $T$ is not the sampling period of the underlying motion system, which is typically much smaller.

Remark 3: In most (sampled-data) extremum-seeking literature, $Q$ reflects the steady-state behavior of the dynamical system. In those cases, the sampling period $T$, or so-called waiting time $T$ [12]-[14], needs to be chosen sufficiently large by the user such that the closed-loop extremum-seeking scheme is robust against inexact measurements of the cost $Q$ due to the transient behavior of the system. Here, $Q$ in (14) actually incorporates the transient behavior of the system, which ultimately determines positioning accuracy. As such, the role of the waiting time $T$ is different here and is conveniently chosen equal to the period time $T$ of the repetitive motion profile.

Remark 4: A common requirement in the extremum-seeking literature is that the input-output mapping $Q$ is independent of initial conditions. Here, the transient behavior is partly determined by the initial conditions, and reinitialization after each setpoint operation is theoretically required for an input-output mapping $Q$ as in (14) to be uniquely defined. Reinitialization for transient performance optimization is also a well-known and commonly accepted requirement in the ILC literature [18], [28].

\section{B. Extremum-Seeking Algorithms}

Let the extremum-seeking algorithm $\Sigma$ be any algorithm that solves the optimization problem of finding the minimum $y^{*}$ of $Q(u)$

$$
y^{*}:=\min _{u} Q(u) .
$$

Within the periodic sampled-data extremum-seeking framework, and depending on the nature of the input-output mapping $Q$, many algorithms from the optimization literature can be employed to solve (19). For example, in [14], the so-called DIRECT and Shubert algorithms (see [29], [30], respectively) are employed to find the global extremum of $Q$. If finding a local minimum suffices or if $Q$ possesses only a single (global) extremum, the classical gradient descent or Newton method can be used (see [31]).

Without loss of generality, we employ here the following gradient descent algorithm to optimize the vector $u$ :

$$
u_{k}=u_{k-1}-\lambda \nabla Q\left(u_{k-1}\right)
$$


with $\lambda$ the optimizer gain. Since $Q$ is unknown, its gradient $\nabla Q$ is unknown. As such, the gradient of $Q$ will be estimated based on finite differences as follows:

$$
\nabla Q(u) \approx \frac{1}{\tau}\left[\begin{array}{c}
Q\left(u+\tau d_{1}\right)-Q(u) \\
\vdots \\
Q\left(u+\tau d_{p}\right)-Q(u)
\end{array}\right]
$$

where $\tau$ is the step size of the gradient estimator and $d_{j}$ with $j=1, \ldots, p$ are dither signals, i.e., vectors where the $j$ th element is equal to one, and all other elements are zero. Moreover, $d_{0}$ denotes a zero vector. Dithering needs to be done in a sequential manner to acquire the elements in (21).

In order to improve the accuracy of the gradient estimation in the presence of, e.g., measurement noise, we include the possibility to repeat each iteration $q$ times and average the measured costs over the $q$ repetitions. As such, the gradient descent algorithm in (20) and the sequence of dithers to obtain the approximate gradient in (21) can be implemented through the following extremum-seeking algorithm:

$$
u_{k}= \begin{cases}u_{k-n}+\tau d_{\frac{n-m}{q}} & \text { if } n \neq 0 \\ u_{k-q(p+1)}-\lambda \nabla Q\left(u_{k-q(p+1)}\right) & \text { if } n=0\end{cases}
$$

for all $k=1,2, \ldots$, with $n=\bmod (k, q(p+1)) \in \mathbb{N}$ and $m=$ $\bmod (k, q) \in \mathbb{N}$, initial input $u_{0}, p$ to-be-optimized parameters, $q$ repeated iterations, and

$$
\begin{aligned}
\nabla Q\left(u_{k-q(p+1)}\right) \\
=\frac{1}{q \tau}\left[\begin{array}{c}
\sum_{j=0}^{q-1}\left(Q\left(u_{k-q p+j}\right)-Q\left(u_{k-q(p+1)+j}\right)\right) \\
\vdots \\
\sum_{j=0}^{q-1}\left(Q\left(u_{k-q+j}\right)-Q\left(u_{k-q(p+1)+j}\right)\right)
\end{array}\right] .
\end{aligned}
$$

Note that the case $n=0$ in (22) implements an update of the control signal $u$.

We emphasize that the input-output map $Q$ is generally unknown, which is exactly the main motivation to adopt a data-based performance optimization approach such as ESC. Under the assumption that $Q$ is (locally) convex and the fact that solutions are bounded on the considered finite-time interval by Proposition 1, employing the abovementioned gradient descent method results in a decrease of the (bounded) cost between iterations. By design of $Q$ in (14), a decrease of the position error between trials is then realized, which, in turn, implies convergence of the position response to (a small neighborhood of) the setpoint. In addition, the proposed design of $k_{i}(t)$ warrants stability since the system reduces to a PD-controlled mass with friction on $\left[T_{B}, T\right]$, for which the origin is known to be Lyapunov stable (see [10, Sec. 3]).

\section{IMPLEMENTATION SUMMARY}

In this section, a brief summary of the design procedure for achieving high-accuracy repetitive setpoint positioning of frictional motion systems is provided. We would like to emphasize that in this work, we have mainly focused on frictional motion systems, stabilized by a linear PID feedback controller, that suffer from the Stribeck effect. However, the following design procedure can also prove useful to improve transient behavior of motion control systems performing repetitive positioning tasks in the absence of (severe) frictional effects and by iteratively adapting (other) performance relevant tunable parameters.

A priori, we assume that a (stabilizing) linear PID controller has been designed for the (unknown) frictional motion system at hand is and its settings are assumed to be known. Typically, such a linear PID controller has been designed based on measured frequency response functions, which neglect frictional effects such as the Stribeck effect. The (Stribeck) friction characteristic at hand is considered to be unknown. The system is required to perform a known $T$-repetitive motion with a desired (step) reference from the initial position 0 to position $r$. Moreover, the standstill time instance $T_{B}$ and the period time $T$ are provided, which define the transient time window $\left[0, T_{B}\right)$ during which the system is allowed to move from 0 to $r$, and the standstill time window $\left[T_{B}, T\right]$, during which standstill at $r$ is required. To achieve high-accuracy setpoint positioning in this motion setting, the following design procedure can be employed.

1) Design a cost function [see (6) and implemented through (15)] that captures the desired performance, and define appropriate weights or penalties $w_{1}$ and $w_{2}$, associated with the transient time window $\left[0, T_{B}\right)$, and the standstill time window $\left[T_{B}, T\right]$, respectively.

2) Replace the linear PID feedback controller in (3) by the time-varying PID feedback controller in (8) with switching function $\varsigma(t)$ in (12).

a) Use the same values for the parameters $k_{p}$ and $k_{d}$.

b) Parameterize the time-varying integrator gain $k_{i}(t)$ by a set of basis functions [see (9)] and choose the type of basis functions (see Example 1 or 2).

c) Given the choice of basis functions, choose the number of basis functions $b$. This determines the size of the to-be-designed parameter vector $v \in$ $\mathbb{R}^{n_{b}}$, with $n_{v}$ the number of elements. A large amount of basis functions allows a more flexible design for $k_{i}(t)$; however, it requires more parameters to be tuned later on. This may yield a more complex objective function with many local minima which is not desired when using gradient-based optimization methods. Moreover, visualization of the objective function becomes increasingly difficult or impossible with $p \geq 3$, and more parameters to-be-designed typically lead to slower convergence which can be undesired.

3) Decompose the to-be-designed parameter vector $v$ as $v=v_{0}+C u$.

a) Design the initial parameter vector $v_{0} \in \mathbb{R}^{n_{v}}$, having $n_{v}$ components. A good choice for the first element of $v_{0}$ would be to use the value for $k_{i}$ used in the linear case. A value of 0 for the elements that correspond to the standstill time window is a good choice as well.

b) Select the specific parameters to-be-optimized through the user-defined selection matrix $C \in \mathbb{R}^{n_{D} \times p}$, with $p$ the amount of parameters to-be-optimized, and $u$ the vector of 
to-be-optimized parameters by the extremum-seeking algorithm.

4) Implement the extremum-seeking controller, i.e., a $\mathrm{ZOH}$ element in (17), a periodic sampler in (18), and an extremum-seeking algorithm to optimize the input vector $u$. The extremum-seeking algorithm can be of the gradient-descent type, such as the one in (22) and (23). Here, the step size $\tau \in \mathbb{R}_{>0}$, the optimizer gain $\lambda \in \mathbb{R}_{>0}$, and the number of repetitions of the same iteration $q \in \mathbb{N}_{>0}$ must be defined by the user.

In Section VI, this design procedure has been employed to demonstrate the working principle and the effectiveness of the proposed PID-based learning controller on an industrial nanopositioning stage.

\section{PID-BASED LEARNING CONTROL FOR AN INDUSTRIAL NANOPOSITIONING MOTION STAGE}

In this section, we demonstrate the working principle and the effectiveness of the proposed PID-based learning controller on an industrial nanopositioning stage. The considered stage represents a sample manipulation stage of an electron microscope [32], exhibiting significant and unknown frictional effects.

\section{A. System Description}

The experimental setup is presented in Fig. 5. The setup consists of a Maxon RE25 DC servo motor (1) connected to a spindle (2) via a coupling (3) that is stiff in the rotational direction while being flexible in the translational direction. The spindle drives a nut (4), transforming the rotary motion of the spindle to a translational motion of the attached carriage (5), with a ratio of $7.96 \times 10^{-5} \mathrm{~m} / \mathrm{rad}$. A coiled spring (8) connects the carriage to the fixed world to eliminate any backlash between the spindle and the nut. The position of the carriage is measured by a linear Renishaw encoder (6) with a resolution of $1 \mathrm{~nm}$ (and a peak noise level of $4 \mathrm{~nm}$ ).

For frequencies up to $200 \mathrm{~Hz}$, the system dynamics can be well described by the model in (2). The mass $m=172.6 \mathrm{~kg}$ consists of the transformed (rotational) inertia of the motor and the spindle (with an equivalent mass of $171 \mathrm{~kg}$ ) and of the mass of the carriage $(1.6 \mathrm{~kg})$.

The friction characteristic for $\Phi$ in (2) is dominated by the bearings supporting the motor axis and the spindle (see (7) in Fig. 5) and by the contact between the spindle and the nut (4). The latter contact is lubricated, which induces a Stribeck effect. Since the system is rigid and behaves as a single mass for frequencies up to $200 \mathrm{~Hz}$, these friction forces can be summed up to provide a single net friction characteristic as $\Phi$ in (2).

Remark 1: The experimental setup is the same as the setup in $[9$, Sec. 5], where dominantly Coulomb and viscous friction was present. For the experimental study in this article, a different carriage position and spindle orientation, and different lubrication conditions result in a significant Stribeck effect instead, as shown in Fig. 2 and the experimental results.

According to the standard operation of the nanopositioning stage in an electron microscope, we can only use a higher order

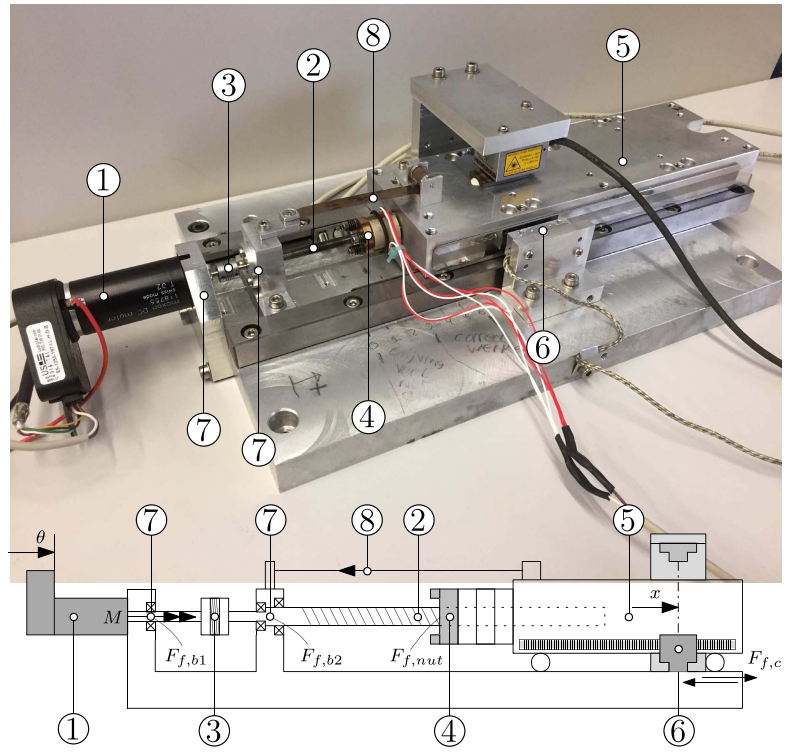

Fig. 5. Industrial nanopositioning motion stage setup. (1): Maxon RE25 DC servo motor. (2): spindle. (3): coupling. (4): nut. (5): carriage. (6): linear Renishaw encoder. (7): bearings. (8): coiled spring.

reference trajectory. Therefore, the step reference $r=1 \mathrm{~mm}$ is mimicked by a fast third-order reference trajectory. We require the carriage to be in standstill at $r=1 \mathrm{~mm}$ at $T_{B}=1.5 \mathrm{~s}$, and the setpoint operation ends at $T=3 \mathrm{~s}$. After each setpoint operation, the system is reinitialized to its starting position $x_{1}=0 \mathrm{~mm}$ using an internal homing procedure.

\section{B. Controller Settings and ESC-Based Optimal Tuning}

The design of the PID-based controller with time-varying integrator gain used in the experiments is discussed in Section III. First, the PID-controller gains are tuned using linear loop-shaping techniques [33], resulting in $k_{p}=10^{7} \mathrm{~N} / \mathrm{m}$ and $k_{d}=2 \times 10^{3} \mathrm{Ns} / \mathrm{m}$. The time-varying integrator gain is parameterized by (9) with $b=6$ linear spline basis functions as in Example 2, from which follows that $t_{s}=(T / b)=0.5 \mathrm{~s}$. We select $p=2$ parameter to-be-optimized. The parameter vector $v=v_{0}+C u$, with initial parameter vector $v_{0}=$ $\left[\begin{array}{llllllll}1 \cdot 1 & 10^{8} & 0 & 0 & 0 & 0 & 0 & 0\end{array}\right]^{\top}$, and a selection matrix

$$
C=\left[\begin{array}{lllllll}
0 & 1 & 0 & 0 & 0 & 0 & 0 \\
0 & 0 & 1 & 0 & 0 & 0 & 0
\end{array}\right]^{\top} .
$$

The first element of vector $v_{0}$ is equal to the constant integrator gain of a classical PID controller, as obtained by the loop-shaping procedure (Fig. 2 shows the measured responses with these settings). The vector $u \in \mathbb{R}^{2 \times 1}$ will be determined by the ESC algorithm discussed in Section IV-B, and the performance of the control system in the sense of (14) depends on the value of these parameters.

For the current case study, we focus on setpoint accuracy rather than transient performance. Therefore, we define the system's performance by the objective function $Q$ in (14) and implemented by (15), where we have taken $w_{1}=0$ and $w_{2}=$ $1 \times 10^{8}$ in (16). Moreover, we augment $Q$ with a logarithmic barrier function [34] in order to restrict the values of the 


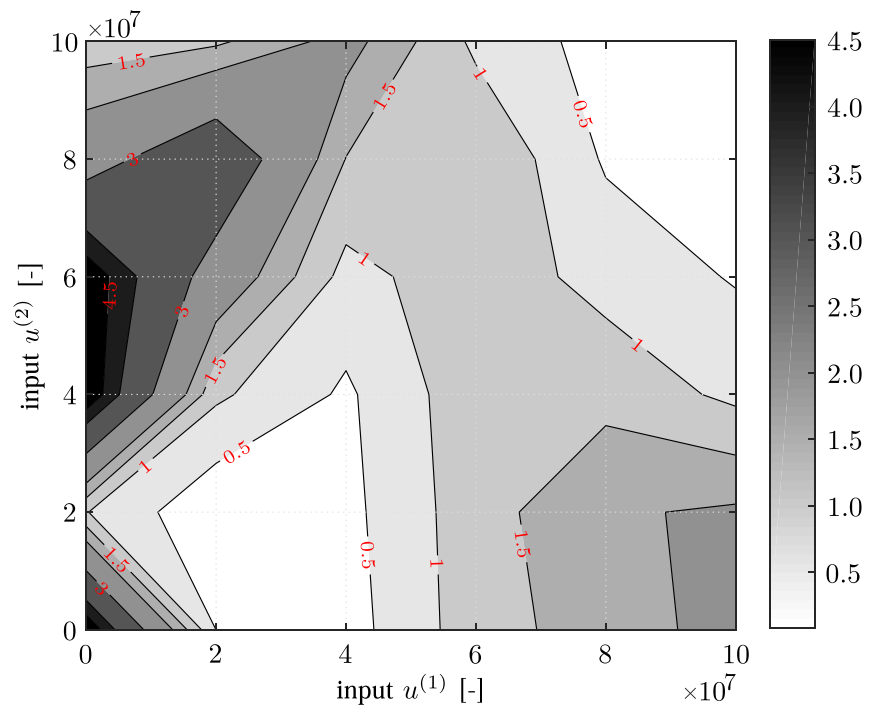

Fig. 6. Contour plot of an experimentally obtained input-output mapping $\tilde{Q}(u)$, which shows two regions where $\tilde{Q}(u)$ is small, indicating integrator gain settings that yield accurate setpoint positioning.

parameter values found by the extremum-seeking controller such that $k_{i}(t)$ remains bounded for all $t \in[0, T]$. In particular, $k_{i}(t)$ then satisfies $\underline{k}_{i} \leq k_{i}(t) \leq \bar{k}_{i}$, with $\underline{k}_{i}=-0.2 \times 10^{8}$, and $\bar{k}_{i}=1.2 \times 10^{8}$. The augmented objective function is then given by

$$
\tilde{Q}(u):=Q(u)+\mu B(u)
$$

with $Q(u)$ as in (14), $\mu=1 \times 10^{-4}$ the barrier parameter, and the logarithmic barrier function $B$ given by

$$
B(u):=-\sum_{i=1}^{4} \log \left(-G_{i}(u)\right)
$$

with $G_{1}(u)=u^{(1)}-\bar{k}_{i}, G_{2}(u)=\underline{k}_{i}-u^{(1)}, G_{3}(u)=u^{(2)}-\bar{k}_{i}$, and $G_{4}(u)=\underline{k}_{i}-u^{(2)}$.

To minimize $\tilde{Q}$, we employ the gradient descent algorithm as discussed in Section IV-B, with $q=3, p=2$, step size $\tau=0.25 \times 10^{7}$, and gain $\lambda=2 \times 10^{16}$, unless stated otherwise. As such, for each controller update by the gradient descent algorithm, $q(p+1)=9$ setpoint operations of $T=3 \mathrm{~s}$ are required.

\section{Experimental Results}

1) Static Input-Output Mapping: The dependence of the achievable setpoint accuracy, captured by the performance metric $\tilde{Q}$ in (24) to be minimized, on the vector $u$ is depicted by means of an measured input-output mapping $\tilde{Q}$ in Fig. 6 . We use this mapping to verify the time-domain results presented later on. Two regions are observed where $\tilde{Q}$ is small, indicating integrator gain settings that can lead to a high setpoint accuracy. Such an input-output mapping, however, is in general time-consuming to obtain and can vary (slowly) over time and can vary from machine to machine. Hence, such an offline, brute-force approach to performance optimization is not feasible in practice. Therefore, the optimal parameter
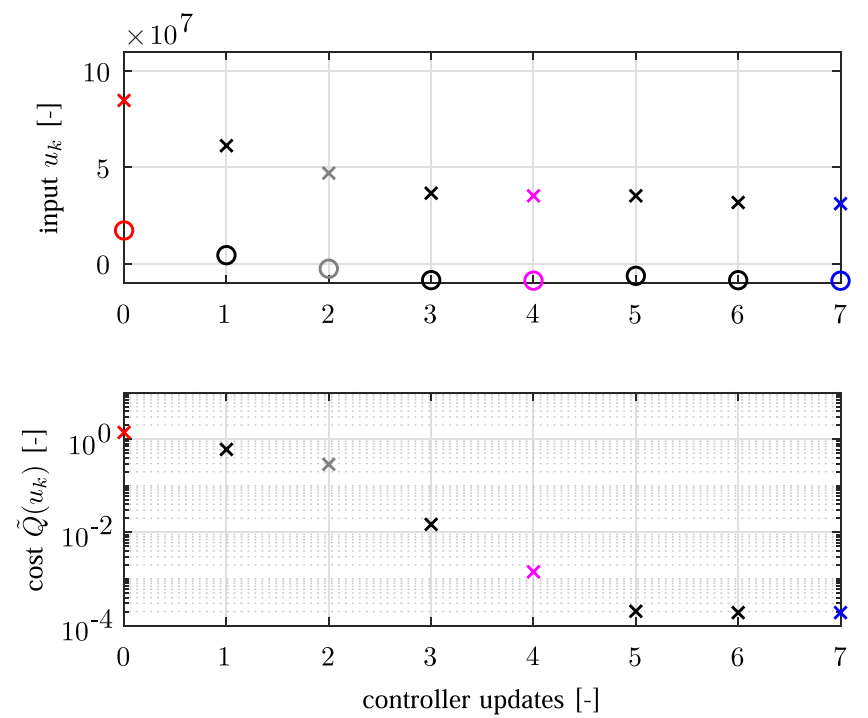

Fig. 7. Experimental results of the PID-based learning controller applied to the industrial nanopositioning motion stage, illustrating the minimization of the augmented performance cost $\tilde{Q}$ (bottom) and the corresponding parameter vector $u_{k}$ (top) (' $\times$ ' and ' $\circ$ ' denote the first and second parameters of the vector $u_{k}$, respectively), as a function of the controller updates. Fig. 8 shows the setpoint error, and the corresponding time-varying integrator gain $k_{i}(t)$, and control force $u_{c}$, corresponding to the initial parameter setting $u_{0}=$ $\left[\begin{array}{ll}0.85 & 0.175\end{array}\right]^{\top}(--)$, the second $(-\cdots)$, fourth $(\cdots)$, and seventh $\left(-\frac{}{}\right)$ extremum-seeking controller update.

settings are iteratively obtained by the online ESC algorithm presented in Section IV-B, solely based on real-time output measurements.

2) Time-Domain Results Obtained by Extremum Seeking: Consider Fig. 7, which shows the measured augmented performance cost $\tilde{Q}\left(u_{k}\right)$ as in (24) and the corresponding vector of parameters $u_{k}$ as determined by the extremum-seeking controller, as a function of the controller updates, starting with initial parameter vector $u_{0}=[0.85,0.175]^{\top} \cdot 10^{8}$. Moreover, Fig. 8 shows the setpoint error $e(t)$, the corresponding time-varying integrator gain design $k_{i}(t)$, and the resulting control force $u_{c}$ for four different controller updates (final and three intermediate). It can be observed that limit cycling is indeed prevented since $k_{i}(t)=0$ for all $t \in\left[T_{B}, T\right]$, and we only observe one interval of stick (during the standstill time window, as desired). Moreover, the extremum-seeking controller iteratively finds controller parameters $u_{k}$ that result in a relatively small time-varying integrator gain design $k_{i}(t)$ on $t \in[0,1.5)$, yielding a position error in the range of 4-6 nm, depicted by $(-)$. In contrast, the classical PID controller for this particular measurement yields an absolute error of about $100 \mathrm{~nm}$ on the same time interval (see Fig. 2) and does not provide robustness during the standstill time window. This clearly illustrates the performance benefits of the proposed PID-based learning controller in terms of the ability to cope with Stribeck friction and achieving superior setpoint positioning accuracy. The parameter evolution of this experiment is visualized by (---) in the input-output mapping in Fig. 9.

Another interesting optimization experiment and resulting time-domain response is the one shown in Fig. 10 

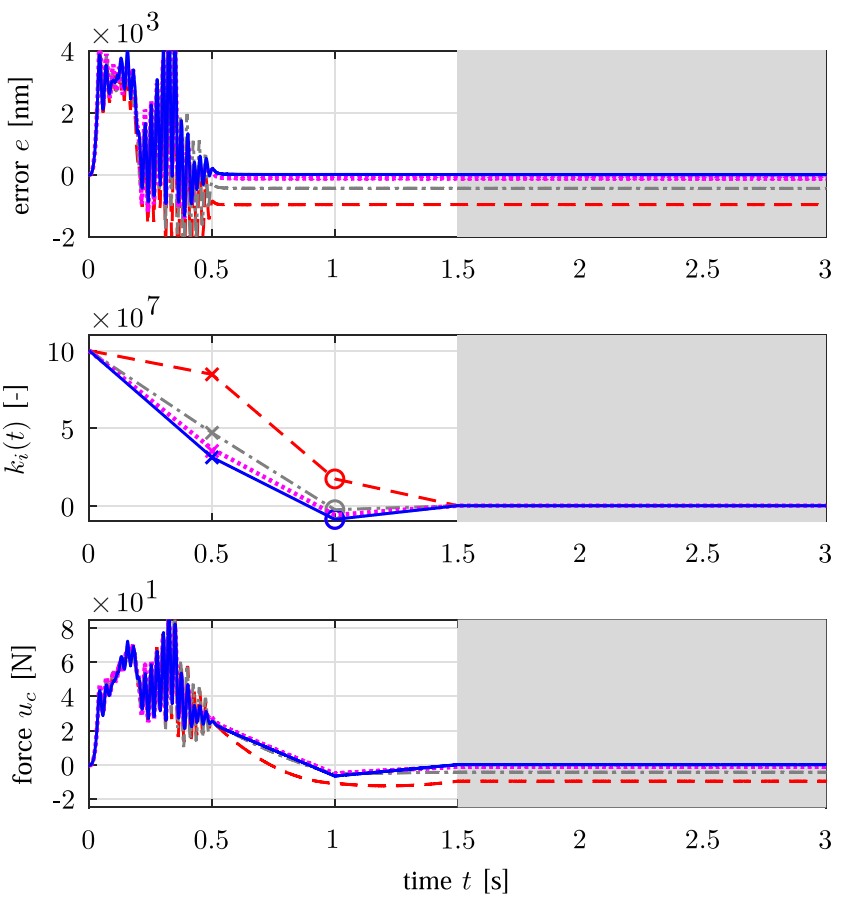

Fig. 8. Experimental results of the PID-based learning controller applied to the industrial nanopositioning motion stage, illustrating the optimization of the setpoint error (for $t>1.5$ ) by adaptation of $k_{i}(t)$. The error and corresponding $k_{i}(t)$ and $u_{c}$ are shown after the initial parameter setting $u_{0}=[0.85,0.175]^{\top} \cdot 10^{8}(--)$, the second $(-\cdots)$, fourth $(\ldots$.$) , and$ seventh ( - ) extremum-seeking controller update. The shaded area denotes the standstill time window. The achieved accuracy for $t>1.5$ after the seventh update is about $4-6 \mathrm{~nm}$.

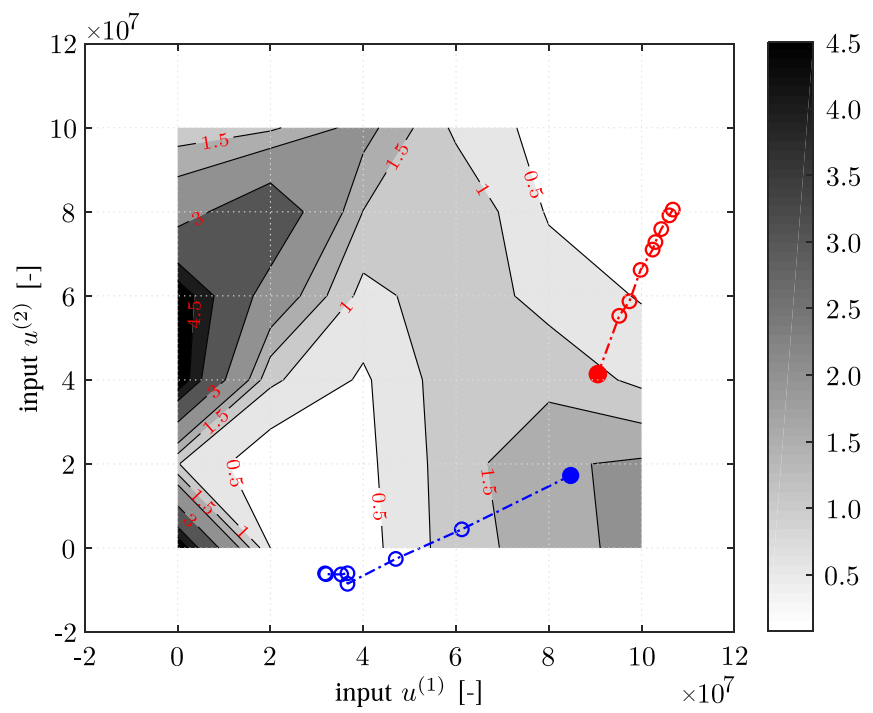

Fig. 9. Input-output mapping $\tilde{Q}(u)$. The figure shows the convergence to optimal integrator gain settings starting from two different initial conditions $u_{0}$, namely, $u_{0}=\left[\begin{array}{ll}0.85 & 0.175\end{array}\right]^{\top} \cdot 10^{8}(\ldots)$ and $u_{0}=\left[\begin{array}{ll}0.9 & 0.4\end{array}\right]^{\top} \cdot 10^{8}(\mathbf{- . -})$. The starting point of both trajectories is denoted by the solid dot.

by (-), which shows the time-domain results after the final extremum-seeking controller update, when using initial parameter vector $u_{0}=[0.9,0.4]^{\top} \cdot 10^{8}$. Again, the parameter evolution of this experiment is visualized by (..-) in the input-output mapping in Fig. 9. The parameters now converge toward the local minimum in the top-right corner of the input-output mapping in Fig. 9. The existence of this particular (local) minimum can be explained by considering
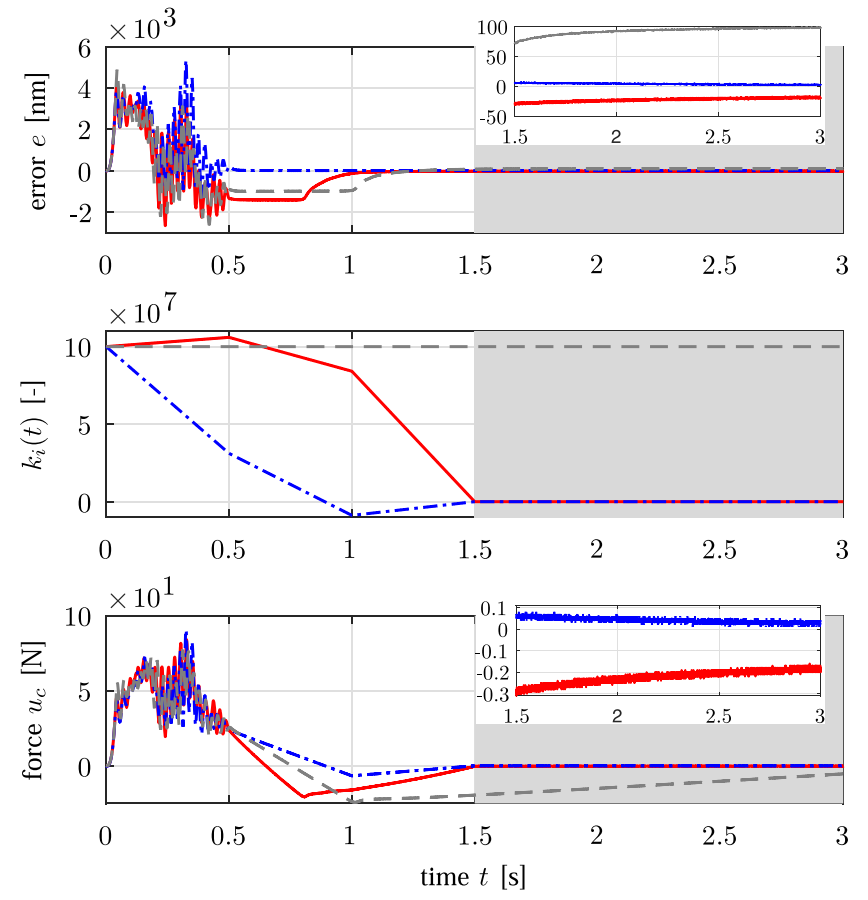

Fig. 10. Experimental results of the PID-based learning controller applied to the industrial nanopositioning motion stage. The figure illustrates the resulting setpoint errors $e(t)$ and the corresponding $k_{i}(t)$ and $u_{c}(t)$ after the final extremum-seeking controller update, for two different experiments, depicted by ( -.-) and (-). The results using the linear PID control are depicted by (_. . -) for comparison. The shaded area denotes the standstill time window.

the top two subplots of Fig. 10, depicting the position error and time-varying integrator gain design $k_{i}(t)$, respectively. Due to the relatively large time-varying integrator gain $k_{i}(t)$ for $t \in[0,1.5)$ obtained by the extremum-seeking controller and depicted by (-), the associated integral action during the transient results in a significant overshoot of the setpoint. The significant overshoot can be attributed to the weight $w_{1}=0$, chosen during the transient time window, which implies that large transients are not penalized. The system then arrives in a stick phase, where the control force is built up by the integrator action. Eventually, the system slips and, due to the Stribeck effect in combination with the decreasing integrator gain, the system arrives in a stick phase again close to the setpoint. For comparison, Fig. 10 shows the experimental results of the linear PID feedback controller, which shows a significant setpoint error of $100 \mathrm{~nm}$ at $t=3 \mathrm{~s}$.

Remark 5: From the zoomed-in view in Fig. 10, it follows that the system with time-varying integrator gains is not completely at rest during the standstill time window $t \in$ $[1.5,3]$, during which only PD control is active, while we expect the system to be in stick. This can be attributed to other microscopic frictional effects that play a role. However, these are not treated here as they are beyond the scope of this article.

The experimental results show that the proposed time-varying PID controller results in superior positioning accuracy (compared with classical PID control) and that the extremum-seeking controller successfully finds the optimal tuning of the time-varying integrator gain, regardless of the initial values of $u$, for the unknown frictional situation at hand. 


\section{CONCLUSION}

We have presented a novel time-varying integrator gain design for motion systems with unknown Coulomb and velocity-dependent friction (including the Stribeck effect), performing a repetitive motion profile. The proposed controller is capable of achieving a high positioning accuracy, in contrast to classical PID control, which often leads to limit cycling, i.e., loss of setpoint stability. The time-varying integrator gain is parameterized by linear basis functions, resulting in a continuous control signal. The specific tuning of the time-varying integrator gain, which results in a high setpoint accuracy in the presence of unknown friction, is iteratively obtained by employing a sampled-data extremum-seeking framework. The performance benefits of the proposed control architecture are experimentally demonstrated on a nanopositioning stage in an electron microscope, illustrating its superior performance over classical PID control.

\section{APPENDIX \\ PROOF OF PROPOSITION 1}

Without loss of generality, consider $r=0$ (which implies $e=-x_{1}$ in (8)), and we consider the intervals $\left[0, T_{B}\right)$ and $\left[T_{B}, T\right]$ separately.

By design, we have $\varsigma=1$ on the interval $\left[0, T_{B}\right)$. Then, with state vector $x:=\left[x_{1}, x_{2}, x_{3}\right]^{\top}$, for all $t \in\left[0, T_{B}\right)$, we rewrite the closed-loop system (1), (2), and (8) as

$$
\dot{x} \in A(t) x-e_{2}\left(F_{s} \operatorname{Sign}\left(x_{2}\right)-f\left(x_{2}\right)\right)
$$

with

$$
A(t)=\left[\begin{array}{ccc}
0 & 1 & 0 \\
-k_{p} & -k_{d}-\gamma & -k_{i}(t) \\
1 & 0 & 0
\end{array}\right], \quad e_{2}=\left[\begin{array}{l}
0 \\
1 \\
0
\end{array}\right] .
$$

Define $f_{L}\left(x_{2}\right):=L x_{2}-f\left(x_{2}\right)$, which satisfies $f_{L}\left(x_{2, a}\right) \leq$ $f_{L}\left(x_{2, b}\right)$ for each $x_{2, a}<x_{2, b}$ and $L>0$ by Assumption 1(3), i.e., $f_{L}\left(x_{2}\right)$ is nondecreasing. Next, define $\Phi_{L}\left(x_{2}\right):=$ $F_{s} \operatorname{Sign}\left(x_{2}\right)+f_{L}\left(x_{2}\right)$, and rewrite (26) as

$$
\begin{aligned}
\dot{x} & \in\left[\begin{array}{ccc}
0 & 1 & 0 \\
-k_{p} & L-k_{d}-\gamma & k_{i}(t) \\
-1 & 0 & 0
\end{array}\right] x-e_{2} \Phi_{L}\left(x_{2}\right) \\
& =: A_{L}(t) x-e_{2} \Phi_{L}\left(x_{2}\right) .
\end{aligned}
$$

Existence of solutions ${ }^{1}$ to (28) follows from [35, Sec. 7, Th. 1] because the set-valued mapping in (28) is outer semicontinuous and locally bounded with nonempty compact convex values. Consider then two solutions $x_{a}$ and $x_{b}$ to (28) with $x_{a}(0)=x_{b}(0)$, and define $\delta:=x_{a}-x_{b}$. For almost all $t \in\left[0, T_{B}\right)$

$$
\dot{\delta} \in A_{L}(t) \delta-e_{2}\left(\Phi_{L}\left(x_{2, a}\right)-\Phi_{L}\left(x_{2, b}\right)\right) .
$$

Since $k_{i}(t)$ is bounded by design, there exists $M_{1}>0$ such that $|A(t)| \leq M_{1}$ for all $t \in\left[0, T_{B}\right)$, with $|A(t)|$ the (induced)

\footnotetext{
${ }^{1}$ A solution to (28) is any locally absolutely continuous function $x$ that satisfies (26) for almost all $t \in\left[0, T_{B}\right.$ ).
}

2-norm of matrix $A(t)$. Then, we have

$$
\begin{aligned}
\frac{1}{2} \frac{d}{d t}|\delta|^{2} & \in \delta^{\top} A_{L}(t) \delta+\delta_{2}^{\top}\left(\Phi_{L}\left(x_{2, b}\right)-\Phi_{L}\left(x_{2, a}\right)\right) \\
& \leq M_{1}|\delta|^{2}+\max _{\substack{f_{b} \in \Phi_{L}\left(x_{2, a}(t)-\delta_{2}(t)\right) \\
f_{a} \in \Phi_{L}\left(x_{2, a}(t)\right)}} \delta_{2}\left(f_{b}-f_{a}\right) \\
& =: M_{1}|\delta|^{2}+N(t) .
\end{aligned}
$$

Whether $x_{2, a}(t)$ and $x_{2, a}(t)-\delta_{2}(t)$ are positive, zero, or negative, inspection of all cases reveals that $N(t) \leq 0$ for all $t \in\left[0, T_{B}\right)$ because $f_{L}$ is nondecreasing, which implies that $\Phi_{L}$ is nondecreasing. As a result, (29) satisfies

$$
\frac{1}{2} \frac{d}{d t}|\delta|^{2} \leq M_{1}|\delta(t)|^{2}
$$

for almost all $t \in\left[0, T_{B}\right)$. Then, $\delta(0)=0$ implies $\delta(t)=0$ for all $t \in\left[0, T_{B}\right)$ by standard comparison theorems (see [36, Lemma 3.4]).

On $\left[T_{B}, T\right]$, we have $\dot{x}_{3}=\varsigma x_{1}=0$ because, by design, the switching function $\varsigma=0$ on the considered interval so that $x_{3}(t)=x_{3}\left(T_{B}\right)$ for all $t \in\left[T_{B}, T\right]$. Moreover, $k_{i}(t)=0$ for $t \in\left[T_{B}, T\right]$. With

$$
\begin{aligned}
\dot{x} & \in\left[\begin{array}{ccc}
0 & 1 & 0 \\
-k_{p} & L-k_{d}-\gamma & 0 \\
0 & 0 & 0
\end{array}\right] x-e_{2} \Phi_{L}\left(x_{2}\right) \\
& =: A_{L_{2}}(t) x-e_{2} \Phi_{L}\left(x_{2}\right) .
\end{aligned}
$$

We obtain analogously to the previous case

$$
\frac{1}{2} \frac{d}{d t}|\delta|^{2} \leq M_{2}|\delta(t)|^{2}
$$

for almost all $t \in\left[T_{B}, T\right]$, with $M_{2}:=\lambda\left(A_{L_{2}}\right)$ the largest singular value of $A_{L_{2}}$. Using absolute continuity of solutions, and the fact that $\delta(t)=0$ for all $t \in\left[0, T_{B}\right.$ ) (as established above), we have that $\delta\left(T_{B}\right)=0$, and (32) implies that $\delta(t)=0$ for all $t \in\left[T_{B}, T\right]$. Uniqueness of solutions on $[0, T]$ is then proven.

We now turn to proving that solutions to the closed-loop system remain bounded on $[0, T]$. Let $x_{a}$ be a generic solution to the closed-loop system, with $x_{a}(0) \in K_{1} \mathbb{B}$ and $K_{1} \geq 0$, and take $x_{b}(0)=(0,0,0)$ so that $x_{b}(t)=0$ for all $t \in[0, T]$, and $\delta(0) \in K_{1} \mathbb{B}$. The solutions $x_{a}$ and $x_{b}$ satisfy (30) and (32) on $\left[0, T_{B}\right)$ and $\left[T_{B}, T\right]$, respectively. In both inequalities, the right-hand side is bounded for all $t$ in its domain, which excludes finite escape times for $\delta$ on $[0, T]$. Hence, there exists $K_{2}>0$ such that $\delta(t) \in K_{2} \mathbb{B}$ for all $t \in[0, T]$. Since $x_{b}(t)=0$ for all $t \in[0, T]$, we have $x_{a}(t) \in K_{2} \mathbb{B}$ for all $t \in[0, T]$, which completes the proof.

\section{REFERENCES}

[1] S. J. L. M. van Loon, B. G. B. Hunnekens, W. P. M. H. Heemels, N. van de Wouw, and H. Nijmeijer, "Split-path nonlinear integral control for transient performance improvement," Automatica, vol. 66, pp. 262-270, Apr. 2016.

[2] H. Butler, "Position control in lithographic equipment," IEEE Control Syst. Mag., vol. 31, no. 5, pp. 28-47, Oct. 2011.

[3] C. Makkar, G. Hu, W. G. Sawyer, and W. E. Dixon, "Lyapunov-based tracking control in the presence of uncertain nonlinear parameterizable friction," IEEE Trans. Autom. Control, vol. 52, no. 10, pp. 1988-1994, Oct. 2007 
[4] L. Freidovich, A. Robertsson, A. Shiriaev, and R. Johansson, "LuGremodel-based friction compensation," IEEE Trans. Control Syst. Technol., vol. 18 , no. 1 , pp. 194-200, Jan. 2010

[5] N. van de Wouw and R. I. Leine, "Robust impulsive control of motion systems with uncertain friction," Int. J. Robust Nonlinear Control, vol. 22, no. 4, pp. 369-397, Mar. 2012.

[6] L. Iannelli, K. H. Johansson, U. T. Jönsson, and F. Vasca, "Averaging of nonsmooth systems using dither," Automatica, vol. 42, no. 4, pp. 669-676, Apr. 2006.

[7] G. Bartolini, A. Pisano, E. Punta, and E. Usai, "A survey of applications of second-order sliding mode control to mechanical systems," Int. J. Control, vol. 76, nos. 9-10, pp. 875-892, Jan. 2003.

[8] A. Bisoffi, M. Da Lio, A. R. Teel, and L. Zaccarian, "Global asymptotic stability of a PID control system with Coulomb friction," IEEE Trans. Autom. Control, vol. 63, no. 8, pp. 2654-2661, Aug. 2018.

[9] R. Beerens, A. Bisoffi, L. Zaccarian, W. P. M. H. Heemels, H. Nijmeijer, and N. van de Wouw, "Reset integral control for improved settling of PID-based motion systems with friction," Automatica, vol. 107, pp. 483-492, Sep. 2019.

[10] D. Putra, H. Nijmeijer, and N. van de Wouw, "Analysis of undercompensation and overcompensation of friction in 1DOF mechanical systems," Automatica, vol. 43, no. 8, pp. 1387-1394, Aug. 2007.

[11] R. H. A. Hensen, M. J. G. van de Molengraft, and M. Steinbuch, "Friction induced hunting limit cycles: A comparison between the LuGre and switch friction model," Automatica, vol. 39, no. 12, pp. 2131-2137, Dec. 2003.

[12] A. R. Teel and D. Popovic, "Solving smooth and nonsmooth multivariable extremum seeking problems by the methods of nonlinear programming," in Proc. Amer. Control Conf., vol. 3, Jun. 2001, pp. 2394-2399.

[13] K. Kvaternik and L. Pavel, "Interconnection conditions for the stability of nonlinear sampled-data extremum seeking schemes," in Proc. 50th Conf. Dec. Control Eur. Control Conf., Dec. 2011, pp. 4448-4454.

[14] S. Z. Khong, D. Nešić, Y. Tan, and C. Manzie, "Unified frameworks for sampled-data extremum seeking control: Global optimisation and multiunit systems," Automatica, vol. 49, no. 9, pp. 2720-2733, Sep. 2013.

[15] O. Lequin, M. Gevers, M. Mossberg, E. Bosmans, and L. Triest, "Iterative feedback tuning of PID parameters: Comparison with classical tuning rules," Control Eng. Pract., vol. 11, no. 9, pp. 1023-1033, Sep. 2003.

[16] M. F. Heertjes, B. Van Der Velden, and T. Oomen, "Constrained iterative feedback tuning for robust control of a wafer stage system," IEEE Trans. Control Syst. Technol., vol. 24, no. 1, pp. 56-66, Jan. 2016.

[17] H. Hjalmarsson, M. Gevers, S. Gunnarsson, and O. Lequin, "Iterative feedback tuning: Theory and applications," IEEE Control Syst. Mag., vol. 18 , no. 4 , pp. $26-41$, Aug. 1988 .

[18] D. A. Bristow, M. Tharayil, and A. G. Alleyne, "A survey of iterative learning control," IEEE Control Syst. Mag., vol. 26, no. 3, pp. 96-114, Jun. 2006.

[19] Y. Wang, F. Gao, and F. J. Doyle, "Survey on iterative learning control, repetitive control, and run-to-run control," J. Process Control, vol. 19, no. 10 , pp. 1589-1600, Dec. 2009.

[20] S. Z. Khong, D. Nešić, and M. Krstić, "Iterative learning control based on extremum seeking," Automatica, vol. 66, pp. 238-245, Apr. 2016.

[21] M. Benosman, "Multi-parametric extremum seeking-based iterative feedback gains tuning for nonlinear control," Int. J. Robust Nonlinear Control, vol. 26, no. 18, pp. 4035-4055, Dec. 2016.

[22] N. J. Killingsworth and M. Krstić, "PID tuning using extremum seeking: Online, model-free performance," IEEE Control Syst. Mag., vol. 26, no. 1, pp. 70-79, Feb. 2006.

[23] B. Ren, P. Frihauf, R. J. Rafac, and M. Krstic, "Laser pulse shaping via extremum seeking," Control Eng. Pract., vol. 20, no. 7, pp. 674-683, Jul. 2012.

[24] L. Hazeleger, R. Beerens, and N. van de Wouw, "A sampled-data extremum-seeking approach for accurate setpoint control of motion systems with friction," in Proc. 11th IFAC Symp. Nonlinear Control Syst. (NolCos), 2019, vol. 52, no. 16, pp. 801-806.

[25] J. van de Wijdeven and O. H. Bosgra, "Using basis functions in iterative learning control: Analysis and design theory," Int. J. Control, vol. 83, no. 4, pp. 661-675, Apr. 2010

[26] S. H. van der Meulen, R. L. Tousain, and O. H. Bosgra, "Fixed structure feedforward controller design exploiting iterative trials: Application to a wafer stage and a desktop printer," J. Dyn. Syst., Meas., Control, vol. 130, no. 5, pp. 1-16, Sep. 2008.

[27] J. Bolder and T. Oomen, "Rational basis functions in iterative learning control-with experimental verification on a motion system," IEEE Trans. Control Syst. Technol., vol. 23, no. 2, pp. 722-729, Mar. 2015.
[28] M. Nörrlof and S. Gunnarsson, "Time and frequency domain convergence properties in iterative learning control," Int. J. Control, vol. 75, no. 14, pp. 1114-1126, 2002.

[29] D. R. Jones, C. D. Perttunen, and B. E. Stuckman, "Lipschitzian optimization without the lipschitz constant," J. Optim. Theory Appl., vol. 79 , no. 1 , pp. $157-181$, Oct. 1993

[30] B. O. Shubert, "A sequential method seeking the global maximum of a function," SIAM J. Numer. Anal., vol. 9, no. 3, pp. 379-388, Sep. 1972.

[31] S. Boyd, L. Vandenberghe, Convex Optimization. Cambridge, U.K.: Cambridge Univ. Press, 2004.

[32] Thermo Fisher Scientific. [Online]. Available: https://www.fei.com/ products/

[33] G. F. Franklin, D. Powell, and E. Abbas, Feedback Control of Dynamic Systems. Upper Saddle River, NJ, USA: Prentice-Hall, 2002.

[34] L. Hazeleger, D. Nesic, and N. V. de Wouw, "Sampled-data extremumseeking control for optimization of constrained dynamical systems using barrier function methods," in Proc. IEEE 58th Conf. Decis. Control $(C D C)$, Nice, France, Dec. 2019, pp. 213-219.

[35] A. F. Filippov, Differential Equations With Discontinuous Righthand Sides. Norwell, MA, USA: Kluwer, 1988.

[36] H. K. Khalil, Nonlinear Systems, 3rd ed. Upper Saddle River, NJ, USA: Prentice-Hall, 2002.

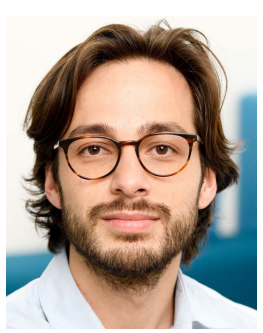

Leroy Hazeleger (Member, IEEE) received the B.Sc. degree in mechanical engineering from the Eindhoven University of Technology, Eindhoven, The Netherlands, in 2012, and the M.Sc. degree in systems and control from the Department of Mechanical Engineering, Eindhoven University of Technology, in 2015. He is currently pursuing the $\mathrm{Ph} . \mathrm{D}$. degree with the Dynamics and Control Group, Eindhoven University of Technology.

His current research interests include nonlinear control systems, data-driven optimization, and extremum-seeking control, with applications to high-performance mechatronic systems.

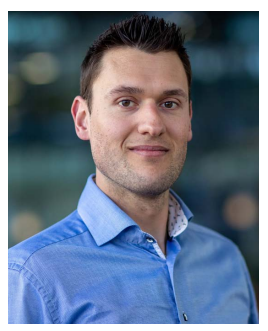

Ruud Beerens received the M.Sc. and Ph.D. degrees in mechanical engineering from the Dynamics and Control Group, Eindhoven University of Technology, Eindhoven, The Netherlands, in 2015 and 2020, respectively.

Since 2020, he has been a Mechatronic Development Engineer in the high-tech semiconductor industry. His current research interests include nonlinear and hybrid control design for high-performance mechatronic systems.

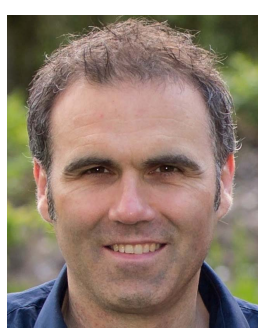

Nathan van de Wouw (Senior Member, IEEE) was born in 1970. He received the M.Sc. degree (Hons.) and the $\mathrm{Ph} . \mathrm{D}$. degree in mechanical engineering from the Eindhoven University of Technology, Eindhoven, The Netherlands, in 1994 and 1999, respectively.

In 2000, he has worked at Philips Applied Technologies, Eindhoven. In 2001, he worked at the Netherlands Organization for Applied Scientific Research (TNO), Delft, The Netherlands. He has held positions as a Visiting Professor at the University of California at Santa Barbara, Santa Barbara, CA, USA, from 2006 to 2007; The University of Melbourne, Melbourne, VIC, Australia, from 2009 to 2010; and the University of Minnesota, Minneapolis, MN, USA, from 2012 to 2013. He was a (part-time) Full Professor with the Delft University of Technology, Delft, from 2015 to 2019. He is currently a Full Professor with the Mechanical Engineering Department, Eindhoven University of Technology. He is also an Adjunct Full Professor with the University of Minnesota. He has published the books Uniform Output Regulation of Nonlinear Systems: A convergent Dynamics Approach with A. V. Pavlov and $\mathrm{H}$. Nijmeijer (Birkhauser, 2005) and Stability and Convergence of Mechanical Systems with Unilateral Constraints with R. I. Leine (Springer-Verlag, 2008). His current research interests include modeling, model reduction, analysis, and control of nonlinear/hybrid and delay systems, with applications to vehicular platooning, high-tech systems, resource exploration, smart energy systems, and networked control systems.

Dr. van de Wouw received the IEEE Control Systems Technology Award for the development and application of variable-gain control techniques for high-performance motion systems in 2015. He is also an Associate Editor of Automatica and the IEEE TRANSACTIONS ON CONTROL SYSTEMS TECHNOLOGY 